too paringly; and allowance must be made for this, for occasionally we had to wait for the arrival of a fresh supply. Another case of railway smash of the foot (Syme's ampatation also) which was not healing kindly (Listerism having been relinquished on account of putrefaction), was treated with boroglyceride, and although healing was neither interfered with nor accelerated, yet smell was unquestionably kept down. Several wounds, the result of either accident or surgical operation, have also been treated with boroglyceride with satisfactory results, and the conclusions I have arrived at so far are as follows: For open wounds or wounds with open cavities boroglyceride in solution will be found to be a non-irritating and powerful antiseptic, in no way interfering with natural healing processes, and in no degree troubling the skin around a wound but where shut cavities exist, as in stumps after amputation, although syringing with the solution be adopted, yet the discharge collecting within is not kept sweet. I feel sure that much remains to be done to develop the use of this new antiseptic, which, on account of its non-irritating properties, will prove of much value in the treatment of wounds. To claim for any antiseptic a value beyond one's experience would be to court distrust, and I prefer to speak hopefully rather than positively.

Mr. Barwell's paper in THE LANCET of May 13th certainly supports our experience of the value of boroglyceride, but we have not succeeded in preventing rises of temperature, any more than we have succeeded in obtaining union without one drop of pus, neither have we seen remarkable results as to healing by first intention; in fact, the results may seem to be nil. I think the use of stronger solution than I to 40 or 1 to 20 may be tried, and the lint around the wound should be kept constantly moistened with the solution. As far as I am aware boroglyceride is innocuous, a matter of great moment, when one recollects how many patients have been poisoned by doses of carbolic acid by inadvertence, how many untoward symptoms have been put down to the use of the carbolic spray and absorption of carbolic acid.

I hope on a future occasion to add more to this brief note, which must conctude with an acknowledgment of thanks to Dr. Walker, who manufactured the boroglyceride himself. Carlisle.

\section{THE DIFFERENTIAL DIAGNOSIS BETWEEN HYSTERICAL PARALYSIS AND POLIO- MYELITIS ANTERIOR.}

\section{BY A. HUGHES BENNETT, M.D.,}

PHYSICIAN TO THE HOSPITAL FOR EPILEPSY AND PARALYSIS, REGENT'S-PARK; AND ASSISTANT-PHYSICIAN TO THE WESTMINSTER HOSPITAL.

IN the experience of every practitioner there must have occurred cases of paralysis in women upon which he has been called upon to decide whether the affection was due to serious organic disease of the nervous system, or to that more protean disorder to which we give the name of hysteria. In many such instances there is often the greatest possible difficulty in arriving at a satisfactory conclusion, and the perplexity of the medical attendant is proportionate to the responsibility he assumes in expressing an opinion, and the important issues upon which his verdict depends. That grave errors in judgment on this complicated problem have frequently been made, must be within the knowledge of all, resulting in misfortune to the patient, discredit to the profession, and a triumphant harvest to the charlatan.

Polio-myelitis anterior, or, as it is more commonly called, "infantile paralysis," is more especially liable to be confounded with hysteria, as the symptoms and progress of the case are very similar in hoth. The paralysis resulting from this serious organic disease of the cord attacks the adult, both in its acute and chrovic forms, much mo:e frequent'y than is generally believed. It is obvious that to make a clear distinction between these two disorders is of the highest imp rtance, as the treatment suitable for the benefit of the one would be useless, or even injurious, if applied to the otber. A consideration of this question appears at the present time to be worthy of discussion, as recently there has been a revival of certain energetic measures in the treatment of paralysis of a supposed func- tional and emotional origin; and Drs. Weir Mitchell, Hlar. fair, and others have recorded successful cases of great interest and importance. Although it is admitted that certain procedures were followed by the most satisfactor results when employed by competent physicians, in properly selected cases, it must equally be granted that the same treatment would in different instances be followed by the most disastrous effects. The writer has not infrenuently seen young women condemned to a couch for years, supposed to be suffering from spinal or uterine affection, which a little energy and determination on the part of the physician would have cured in a few weeks. On the other hand, not les often has be met with unfortunates who were scoffed at and otherwise ill-rreated as being nervous and hysterical, o imagined to be exaggerating or feigning disease, when in reality they were suffering from an organic lesion of the ner vous system. This confusion has arisen from the extreme difficulty in many cases of accurately diagnosing between paralysis, arising on the one band from organic degeneration of nervous structure, and on the other as a result of emotional or so-called hysterical causes. It is assumed in text-books and by the profession generally that the differentiation be tween these is easily defined, but as a matter of fact eren when the greatest care is taken this is not always the case, and after the fullest investigation much doubt remains.

With the view of illustrating this position let us take a case which not infrequently occurs in practice. A youn: woman suddenly or gradually becomes paralysed in the lower extremities. This may, in a few days, weeks, or months, in different cases, become complete or may remain partial. There is no loss of sensation, no muscular rigidity, no cerebral disturbances, or any affection of the blaider or rectum. The patient's general health may be robust, or it may be delicate. She may be of emotional and brsterica temperament, or, on the contrary, of a calm and well. balanced disposition. At first there is no muscular wasting but as the disease becomes chronic the limbs mar, or may not, diminish in size. The entire extremity may he affected, or only certain groups of muscles. Fivally, the disease may partially or entirely recover, or may remain almost unchanged for years.

With such a clinical picture before us we have to ask What is the diagnosis of such a paralysis? The description applies with equal fidelity to either bysteria or polio-myelitis, and by depending on symptoms alone it is in such doubtful cases thet the decision of the question seems to depend on the accidental fancy of the medical attendant, and which so frequently result in an erroneous view being taken of the case. It i granted that the problem is a difficult one, and even a careful analysis of the symptoms does not appear greatly to assist its solution. The patient when attacked with the paralysis may be in perfect healh, or the reverse; she may, or may not, be hysterical ; there may be some assignable cause, or not. In all these circumstances the loss of motion may be either due to polio-myelitis or hysteria, as the former occurs with equal frequency in the robust and in the delicate and the latter as often appears in apparintly the most healthy persons as in the most nervous; the first is as likely to be present in hysterical subjects as in the reverse, and the second may follow a cold, injury, or other supposed origin of organic disease. In short, although it is true that hysterical paralysis frequently occurs in persons evidencing other nervous or emotional symptoms, these are by no means necessary to establish the diagnosis, as some of the most intractable forms of this affection have occurred in wonen of apparently phlegmatic temperament and well-balanced minds. Again, polio-myelitis is as likely to attack the hr. terical subject as any other, and thus further complicate the inquiry. The paralysis appearing suddenly, or very gradu. ally, applies equally to polio-myelitis or hysteria. As in the acute form of the first, the loss of motion may ensue in a fer hours, and in its chronic form it may proceed rery slowly and insidiously. In both the process may be partial or complete. It may attack one or more limls, or only part of an extremity; indeed, special muscles. or irregular groups of these alone, may be affected. All these particulars equally apply to both forms of pard. lysis under consideration. In each the sensibility ma: be intact, the intelligence and special senses unimpirced, acit the functions of the rectum, bladder, and other organe 0 the body healthy. If muscular atrophy existed to a marke extent at an early period of the disease we might excluit hysteria, but in many instances in this respect there is 
Luthing definite to be observed. The limbs in both forms of rurulysis may for a long time appear of natural size. Musculir wasting, if it exists, may be obscured by an increased deposit of fat, and the hypertrophy of other structures. Agaio, if paralysis has existed for any length of time, the limbs nay become smaller, not necessarily due to degnerative atruphy, but to simple emaciation from functional disuse. Thus, as in paralysis resulting from both hysteria and poliomrelitis, the symptoms and general progress of the disease are in many cases identical, it becomes a serious practical dificulty to definitely determine whether in a given case answering to the description already detailed, it is due to degeneration of the anterior cornua of the cord, or to that less formidable affection, hysteria.

To solve the problem, as in other branches of medicine we must not depend on symptoms alone, but, as far as possible, should bring physical phenomena to our aid for the accurate determination, demonstration, and measurement of facts. Exactly in proportion as we thus supplement our senses, so do our powers of precise research and investigation succeed. Although important advances have been made in other fields of medicine by the utilisation of optics, acoustics, and other practical sciences, as evidenced by the employment of the ophthalmoscope, speculum, stethoscope, thermometer, \&c., in diseases of the nervous system such methods of exact inquiry have hitherto been conspicuous by their absence. In recent times, as this want has been appreciated, endeavours have been made to supply the demand. The most successful of these is the employment of electricity as a diagnostic agent ; and although our knowledge of its properties is not as yet fully developed, we have even now at our disposal such information on the subject as proves of invaluable service in the differentiation of many nervons disorders. It has already been pointed out that by spmptoms alone we are not able, in many cases, to distinguish between paralysis resulting from organic disease of the anterior cornua of the cord and that due to hysteria. When we turn to physical signs, we find that there are at least two which give us definite information on the subject. These are-(1) the reflex actions following cutaneous irritation or percussion of tendons, and (2) the effects of electrical stimulation.

1. The Reflex Actions.-When polio-myelitis involves any reflex are, the reflex motions associated with the track interfered with are destroyed. In the case, therefore, of paralys's of the lower limbs, resulting from this disease, all the cutaneous and tendon reflexes are absent. In hysterical paraysis, on the other hand, these phenomena are not abolished. They are usually normal, and not seldom are actually exaggerated in degree, so that not only are those which naturally exist much increased in intensity, but new reflexes not found in health are developed. Here, then, is a marked distinction between hysteria and polio-myelitis. Although this rule applies generally, it must be admitted that it is not without exception. Reflex acts vary somewhat even in healthy persons, and in rare instances have not been obtainalle, Again, it is just possible that in degeneration of the anterior cornua certain parts of their substance might be preserved, and although paralysis existed, certain reflexes might be elicited. In hysteria also the reflexes are sometines absent for reasons not easily explained, Such cases, lowever, are extremely rare, and the general law holds gool that in paralysis from polio-myelitis the reflexes are impaired or abolished, while in that from hysteria they are normal or increased.

The Electricai Peactions. - These are for the most part definite and conclusive. In acute polio-myelitis the excitability of the affected nerves is very rapioly lost, so that in from a week to tea days after the onset of the paralysis the re.pon:e is altogether abolished to the electric stimulus. Very soon afterwards the muscles are affected, and then ensues all the chiracteristic quantitarive and qualitative changes met with in neuro-museular affections. In the chronic forms of the disease the same takes place, but in a more gradual ard prristessive manner. In hysterical paralysis, on the ot ther haud, the electrical reactions remain practically normal. In chas cases there may be quantitative increase of response, indicatiug lyper-excitability of the nervous system, but the e changes are slight in degree, and there are never qualitative alterations. In very chronic cases also, in which the patient liss been bedridden for years, there may be quantitative diminution of response due to prolonged disuse of the muscles, or to increased resistance to the current from an angmented deposition of subcutaneous fat as the result of want of exercise. In such cases the loss is never complete, and the change is one of degree only, and not of character.

Thus in electricity we have an agent which enables us in the large majority of cases to detect with accuracy and certainty between polio-myelitis and hysteria, and, so far as my own experience has gone, I have never met with a case of the former which did not present some characteristic abnormal reactions, nor an instance of the latter in which these were developed.

It is to be observed that great care and much dexterity and experience are necessary for profitable electrical investigation. It cannot be exactly asserted that an expert alone is necessary for this purpose, but certainly it cannot be satisfactorily conducted except by those who, through knowledge and practice, have overcome the technical and manipulative details necessary for its successful performance. The system of wildly dabbing sponge electrodes on the skin without a definite method, is absolutely useless as a means of deducing trustworthy diagnostic data, and it is the popular use in this way which has hitherto been so fruitless and brought a valuable physical agent into discredit. A novice, ignorant of the use of the stethoscope, wonld hear little or nothing on applying it to the chest, and still less would he deduce those im portant conclusions which the experienced physician would recognise. Precisely in the same way is electricity a means towards an end, which can only be put in successful operation by a practical knowledge of the agent employed, with dexterity and experience in its administration.

It would be out of place here to describe eitber the method of procedure or the particulars of the reactions found in the two forms of paralysis under discussion. These have elsewhere been considered in full detail.

The general conclusions to be drawn from the foregoing remarks are:-(1) That, judging from the history, symptoms, and progress of the case, in a large number of iustances it is difficult or impossible to diagnose between paralysis from hysteria or from polio-myelitis; (2) that a correct differentiation between them is of the highest importance, as the treatment successful in the one is useless or may be injurious in the other; (3) that in order to arrive at a true diagnosis, we must as far as posaible apply physical agents in their investigation (4) that in the diseases under consideration the conditions of the reflexes, resulting from physical manipulations, afford us valuable information, although open to certain exceptions ; and (5) that electricity supplies us with an agent which, in the large majority of cases, will definitely enable us to correctly decide whether a given paralysis is due to organic disease of the anterior cornua of the cord, or to that affection to which we apply the term hysteria.

\section{ON BONE-SETTING (SO-CALLED). ${ }^{\mathrm{x}}$}

\section{BY R. DACRE FOX, F.R.C S. EDIY,}

SURGEON TO THE MANCHESTER SOUTHERN FOSPITAL, SURGEON TO THE MANCHESTER POLICE FORCE, CHEF MEDICAL OFFICER TO THE MANCHESTER, SHEFFIELD, AND LINCOLY RAILWAY, ETC.

THIS is the first time in the history of the British Medical Association that so-called bone-setting has been seriously discussed, and I think it is matter for congratulation that we have at last set ourselves impartially to examine the practice, notwithstanding that it is almost exclusively employed by a class of persons who are without our pale. The literature of bone-setting is scauty. Dr. Wharton Hood's handbook, giving an account of the late Mr. Hutton's method of setting free stiff limbs, is, so far as I know, the only attempt at a systematic exposition. There are, in addition, some papers scattered through the various journals, by Mr. Adams, Mr. Howard Marsh, and others, stating their opinion as to what cases are most likely to be benefited by the forcible bending of joints, and giving instances of il results that have followed such treatment in unsuitable cases. For nearly three years I was assistant to the late Mr. Tames Taylor, M.R.C.S., of Whitworth in Lancashire, the last direct descendant of a family that had practised

1 Abridged from a paper read at a discussion before the Surgical Section of the annual meeting of the British Medical Association at Worcester, 1882 